\section{The Significance of Fluctuations in Infection Rates}

\section{To the Editor:}

If I am interpreting the data reported by Haley et al ${ }^{1}$ correctly, their computerized algorithm for cluster detection yields a positive predictive accuracy on the order of $12 \%$ in terms of actual outbreaks detected (1 low-frequency cluster plus 9 statistically significant clusters/82 clusters minus I cluster not investigated for lack of records). Alternatively, it appears that 10 outbreak-confirmed clusters plus 4 systematic surveillance or laboratory error associated clusters $/ 8 \mathrm{l}$ clusters yields a $17 \%$ positive predictive accuracy in terms of systematic problems detected.

This seems much lower than the $33 \%$ overall positive predictive accuracy in our experience. ${ }^{2}$ This could result from differences in the sensitivity and specificity of the two approaches, a difference in the populations to which these approaches are applied, or use of different outbreak threshold limits. Is information available concerning sensitivity and specificity for the algorithm used by the Centers for Disease Control for outbreak detection? Our investigations suggested an upper one-sided threshold level of $\propto=0.02$ to be optimal; is the $p=0.05$ Poison limit used by the CDC one-sided or two-sided?

A simple and reliable method for interpreting the significance of fluctuations in infection rates or frequencies is highly desirable. While positive predictive accuracy is one measure of a system's performance, its sensitivity and specificity determined from its performance in outbreak and nonoutbreak periods seems more important information. Once outbreak threshold limits have been determined, infection frequency can be used for monitoring (rather than infection rate) and use of computers is not essential. In fact, providing interpretive criteria to individual wards could allow decentralization of a surveillance program. This makes an outbreak threshold approach attractive as a screening device in all hospitals, not primarily in larger hospitals as was implied. Further, this approach is analogous to industrial quality assurance methods: it not only detects outbreaks but also provides continuous and timely confirmation that performance is within accepted limits.

Between the nihilists' denial of need for infection surveillance and control programs and the opposite extreme of mandating intensive surveillance in perpetuity, a third strategy should be considered. Once an initial, comprehensive and intensive program has reduced endemic infection rates to levels considered acceptable by an institution's board and administration, an evolution to more cost-effective use of "outbreak threshold surveillance" might then be introduced to maintain performance at those levels. A regional consortium might grow as nearby hospitals decreased their own infection surveillance staffs during this transition; alternatively, hospital epidemiology resources could be assigned to a wider scope of applications as the infection surveillance workload decreased. Either alternative could be more cost-effective than traditional surveillance programs.

\section{REFERENCES}

1. Haley RW, Tenney JH, Lindsey JO, et al: How frequent are outbreaks of nosocomial infection in community hospitals? Infect Control 1985; 6(6):233-236.

2. Birnbaum D: Analysis of hospital infection surveillance data. Infect Control 1984; $5(7): 332-338$.

David Birnbaum, MPH Hospital Epidemiologist Victoria General Hospital Victoria, British Columbia

Dr. Robert Haley responds to $M r$. Birnbaum's comments.*

\section{To the Editor:}

In response to Birnbaum's letter, I will answer his technical questions and then comment on his recommendations. First, for the sake of clarity it should be noted that by the term

*The opinions expressed in this letter are those of the respondent and not an official statement by the Centers for Disease Control. "positive predictive accuracy" Birnbaum is referring to the concept more commonly known in the United States as "predictive value of a positive," or PVP. His estimates of the PVP of our computerized outbreak detection system are correct. I agree with the three possible explanations given for why the PVP of our system (12\% to $17 \%)$ differed from that of his system (33\%). I know of no estimate of the sensitivity and specificity of any computerized system for detecting hospital outbreaks, and I find it difficult to conceive of how one might estimate this practically. Since we were interested only in determining if an infection rate exceeded the expected value, we used a one-tailed test.

Regarding the specific issue of detecting outbreaks, I find Birnbaum's statistical approach interesting and potentially useful. As we showed in our paper, ${ }^{1}$ the majority of outbreaks are recognized by hospital staff who notice an excessive increase in infections without having to calculate rates. An approach that increases the precision of this time-honored process might lead to the increased recognition of the other portion of outbreaks that we found to have gone unnoticed by the hospitals.

Regarding the broader views of surveillance and the future of infection control, Birnbaum and I have fundamentally different views as to what is the best "third strategy." The question of central importance is what approach should infection control personnel take to produce the greatest reduction in nosocomial infection risks. Since, as we and others have found, only $2 \%$ to $3 \%$ of nosocomial infections occur in outbreaks, ${ }^{1,2}$ systems for detecting outbreaks should be only a small part of a hospital's total infection control program, with the vast majority of time, effort and resources being devoted to the prevention of endemic infections. According to the latest information, only a small minority of US hospitals have established programs that can make a significant impact on endemic infection risks. ${ }^{3}$ Thus, rather than reducing the size of hospitals' infection control staffs or depending on regional consortium arrangements, I would strongly recommend that the infection control staff in each hospital 